\title{
LHC phenomenology of natural MSSM with non-universal gaugino masses at the unification scale
}

\author{
Hiroyuki Abe, ${ }^{a}$ Junichiro Kawamura ${ }^{a}$ and Yuji Omura ${ }^{b}$ \\ ${ }^{a}$ Department of Physics, Waseda University, \\ Tokyo 169-8555, Japan \\ ${ }^{b}$ Kobayashi-Maskawa Institute for the Origin of Particles and the Universe (KMI), \\ Nagoya University, \\ Nagoya 464-8602, Japan \\ E-mail: abe@waseda.jp, junichiro-k@ruri.waseda.jp, \\ yujiomur@eken.phys.nagoya-u.ac.jp
}

ABSTRACT: In this letter, we study collider phenomenology in the supersymmetric Standard Model with a certain type of non-universal gaugino masses at the gauge coupling unification scale, motivated by the little hierarchy problem. In this scenario, especially the wino mass is relatively large compared to the gluino mass at the unification scale, and the heavy wino can relax the fine-tuning of the higgsino mass parameter, so-called $\mu$-parameter. Besides, it will enhance the lightest Higgs boson mass due to the relatively large left-right mixing of top squarks through the renormalization group (RG) effect. Then $125 \mathrm{GeV}$ Higgs boson could be accomplished, even if the top squarks are lighter than $1 \mathrm{TeV}$ and the $\mu$ parameter is within a few hundreds $\mathrm{GeV}$. The right-handed top squark tends to be lighter than the other sfermions due to the RG runnings, then we focus on the top squark search at the LHC. Since the top squark is almost right-handed and the higgsinos are nearly degenerate, $2 b+E_{T}^{\text {miss }}$ channel is the most sensitive to this scenario. We figure out current and expected experimental bounds on the lightest top squark mass and model parameters at the gauge coupling unification scale.

KEYWORDS: Supersymmetry Phenomenology

ArXiv EPRINT: 1505.03729 


\section{Contents}

1 Introduction 1

2 Brief review of NUGM scenario 3

3 Phenomenological features of NUGM scenario 4

4 Top squark search at the LHC $\quad 7$

$\begin{array}{lll}4.1 & \text { Details of the analysis } & 7\end{array}$

4.2 Results of the analysis 8

$\begin{array}{lll}5 & \text { Conclusion } & 10\end{array}$

\section{Introduction}

The Minimal Supersymmetric Standard Model (MSSM) is a good candidate for new physics to be found at the LHC [1]. One of the strong motivations to consider a low-scale supersymmetry (SUSY) is to ensure the stability of the electroweak (EW) scale against huge radiative corrections. The other incentives are to provide suitable candidates for dark matters and its prediction of the gauge coupling unification around $2 \times 10^{16} \mathrm{GeV}$, which indicates the existence of the Grand Unification Theories (GUTs).

The natural explanation of the EW scale requires light supersymmetric particles (sparticles), but there has been no signature of SUSY at the LHC Run-I. Moreover, the observed value of the Standard-Model-like Higgs boson mass might imply heavy sparticles since it requires heavy top squark masses to enhance the Higgs boson mass up to $125 \mathrm{GeV}[2,3]$, by the radiative corrections. Such heavy sparticles bring a fine-tuning problem [4] that can be seen in the minimization condition of the Higgs potential,

$$
m_{Z}^{2} \simeq-2\left|\mu\left(m_{Z}\right)\right|^{2}-2 m_{H_{u}}^{2}\left(m_{Z}\right),
$$

where $\mu\left(m_{Z}\right)$ and $m_{H_{u}}\left(m_{Z}\right)$ are a supersymmetric higgsino mass parameter and a soft SUSY breaking mass for the up-type Higgs boson at the $Z$ boson mass $\left(m_{Z} \simeq 91.2 \mathrm{GeV}\right)$ scale, respectively. The soft SUSY breaking mass $m_{H_{u}}$ relates to the masses of the sparticles through the renormalization groups (RG), so $m_{H_{u}}\left(m_{Z}\right)$ tends to be large if sparticles are heavy. Then the severer tuning between $\mu\left(m_{Z}\right)$ and $m_{H_{u}}\left(m_{Z}\right)$ is generally required to realize the observed $Z$ boson mass. In order to derive the EW scale naturally, $m_{H_{u}}\left(m_{Z}\right)$ should be close to the EW scale as much as possible without any conflicts with the observed Higgs mass.

We have proposed a solution to resolve this problem by taking suitable ratios among gaugino masses at the gauge coupling unification scale (GUT scale) $[5,6]$. In the RG 
correction to $m_{H_{u}}\left(m_{Z}\right)$, there are large contributions from the top squarks dominated by the gluino mass. The gluino mass easily makes the magnitude of $m_{H_{u}}\left(m_{Z}\right)$ considerably larger than the EW scale. In the non-universal gaugino mass (NUGM) scenario, the RG correction from the gluino mass are canceled by those similarly from the bino and wino ones, thus the $m_{H_{u}}\left(m_{Z}\right)$ could be small, even if the top squark masses are relatively high. It is remarkable that such a situation could increase the lightest Higgs boson mass due to the larger top squark left-right mixing, which is compatible with the non-universal gaugino masses for reducing the fine-tuning.

In the NUGM scenario, the relatively heavy bino and wino are preferred: particularly, a ratio of wino to gluino mass, $r_{2} \equiv M_{2}\left(M_{\mathrm{GUT}}\right) / M_{3}\left(M_{\mathrm{GUT}}\right)$, should be in the range, $4 \lesssim r_{2} \lesssim 6$. Such a heavy wino enhances the left-handed top squark mass through the $\mathrm{RG}$ running, while the right-handed sparticles do not receive contributions from the wino mass. Moreover, only the right-handed top squark becomes relatively light due to the RG effect through the top Yukawa coupling. ${ }^{1}$ Aims of this paper are to show some specific features of light sparticles in the NUGM scenario and to investigate current experimental bounds on them at the LHC8 and their expected sensitivities at the LHC14, especially focusing on the lightest top squark searches. Since the naturalness argument requires the small $\mu$-parameter, the lightest supersymmetric particle (LSP) is a higgsino-like neutralino. The second lightest higgsino-like neutralino and the lightest chargino are also light, and their mass differences are very small. Therefore a production cross section of the lightest top squark is sizable, and then top squark decays into both higgsino-like neutralinos and chargino together with top or bottom quarks.

The higgsino search may be also relevant to this low-scale SUSY scenario, although their signals of the chargino decay may be buried under the Standard Model (SM) background due to their nearly degenerate masses. There are experimental searches for the charginos and neutralinos, e.g. refs. [7-9]. In refs. [10-12], the way to search for the higgsino pair production at the LHC has been discussed, where small $\mu$-parameter is achieved by tuning the Higgs soft scalar masses [13]. The higgsino search, however, would not be effective to probe the NUGM scenario. Daughter particles of the heavier higgsino-like states will be too soft to be reconstructed in the detector, since the mass gaps among higgsino-like states are highly suppressed due to the heavy wino and bino. A strategy to search for the degenerate higgsinos is studied in ref. [14]. Note that lifetimes of the higgsinos are not so long that they are observed as disappearing tracks unlike the winos as explored in refs. $[15,16]{ }^{2}$ Moreover, heavier neutralino $\widetilde{\chi}_{3,4}^{0}$ and chargino $\widetilde{\chi}_{2}^{ \pm}$are so heavy that hardly produced even at the LHC14 when the gluino is heavy enough to satisfy its lower bound. Therefore direct neutralino or chargino search will not be efficient to probe the NUGM scenario.

\footnotetext{
${ }^{1}$ The right-handed bottom squark may also be light depending on $\tan \beta$. If $\tan \beta$ is large, the negative contributions of the bottom Yukawa coupling to the bottom squark masses are sizable. We $\operatorname{take} \tan \beta=15$ that enhances the tree-level SM-like Higgs boson mass, but small enough to neglect the bottom Yukawa coupling in this paper.

${ }^{2}$ The mass difference between the lightest chargino and neutralino should be $O(0.1 \mathrm{GeV})$ for enough large lifetime of chargino to be observed as disappearing tracks, while the mass difference is $O(1 \mathrm{GeV})$ for higgsino-like states.
} 
This paper is organized as follows. In section 2, we review the NUGM scenario and explain how much the fine-tuning is relaxed while the Higgs boson mass is around $125 \mathrm{GeV}$ in this scenario. In section 3, specific features of the NUGM scenario is explained. In section 4, we discuss the current and expected experimental bounds on the scenario. Finally, we conclude this paper in section 5 .

\section{Brief review of NUGM scenario}

We review the NUGM scenario based on refs. [5, 6]. The most attractive feature of the NUGM scenario is that a certain type of the non-universal gaugino mass spectrum at the GUT scale can relax the fine-tuning of the $\mu$-parameter and helps to enhance the Higgs boson mass.

The lightest CP-even Higgs boson mass in the MSSM can be approximately written as follows at the one-loop level [17]:

$$
m_{h}^{2} \simeq m_{Z}^{2} \cos ^{2} 2 \beta+\frac{3}{8 \pi^{2}} \frac{m_{t}^{4}}{v^{2}}\left[\log \frac{M_{\mathrm{st}}^{2}}{m_{t}^{2}}+\frac{2 \widetilde{A}_{t}^{2}}{M_{\mathrm{st}}^{2}}\left(1-\frac{\widetilde{A}_{t}^{2}}{12 M_{\mathrm{st}}^{2}}\right)\right],
$$

where $\widetilde{A}_{t} \equiv A_{t}\left(m_{Z}\right)-\mu\left(m_{Z}\right) \cot \beta$ is defined. $\tan \beta$ denotes the ratio of vacuum expectation values (VEVs) of two Higgs bosons: $\tan \beta \equiv\left\langle H_{u}^{0}\right\rangle /\left\langle H_{d}^{0}\right\rangle$. The symbols $m_{t}, M_{\mathrm{st}} \equiv$ $\sqrt{\left|m_{Q_{3}} m_{u_{3}}\right|}$ and $A_{t}$ denote the top quark mass, the top squark mass scale and the leftright mixing of the top squarks, called A-term, respectively, while $m_{u_{3}}$ and $m_{Q_{3}}$ are the soft scalar masses for the right-handed top squark and the left-handed third-generation squark, respectively. The Higgs boson mass is far from the observed value when $M_{\text {st }} \lesssim 1 \mathrm{TeV}$ and the last term is negligibly small. The last term is maximized at $\widetilde{A_{t}} \sim \sqrt{6} M_{\text {st }}$. Thus the relatively large A-term of top squarks is necessary to explain the $125 \mathrm{GeV}$ Higgs boson mass.

The parameters relevant to the Higgs boson mass and the stationary conditions for the EW symmetry breaking are $m_{H_{u}}, m_{u_{3}}, m_{Q_{3}}$ and $A_{t}$. Their values at the EW scale depend on the boundary condition at the GUT scale as follows:

$$
\begin{aligned}
m_{H_{u}}^{2}\left(m_{Z}\right) \simeq & -0.01 M_{1} M_{2}+0.17 M_{2}^{2}-0.05 M_{1} M_{3}-0.20 M_{2} M_{3}-3.09 M_{3}^{2} \\
& +\left(0.02 M_{1}+0.06 M_{2}+0.27 M_{3}-0.07 A_{t}\right) A_{t}+0.59 m_{H_{u}}^{2}-0.41 m_{Q_{3}}^{2}-0.41 m_{U_{3}}^{2} \\
m_{Q_{3}}^{2}\left(m_{Z}\right) \simeq & -0.02 M_{1}^{2}+0.38 M_{2}^{2}-0.02 M_{1} M_{3}-0.07 M_{2} M_{3}+5.63 M_{3}^{2} \\
& +\left(0.02 M_{2}+0.09 M_{3}-0.02 A_{t}\right) A_{t}-0.14 m_{H_{u}}^{2}+0.86 m_{Q_{3}}^{2}-0.14 m_{U_{3}}^{2} \\
m_{u_{3}}^{2}\left(m_{Z}\right) \simeq & 0.07 M_{1}^{2}-0.01 M_{1} M_{2}-0.21 M_{2}^{2}-0.03 M_{1} M_{3}-0.14 M_{2} M_{3}+4.61 M_{3}^{2} \\
& +\left(0.01 M_{1}+0.04 M_{2}+0.18 M_{3}-0.05 A_{t}\right) A_{t}-0.27 m_{H_{u}}^{2}-0.27 m_{Q_{3}}^{2}+0.73 m_{U_{3}}^{2} \\
A_{t}\left(m_{Z}\right) \simeq & -0.04 M_{1}-0.21 M_{2}-1.90 M_{3}+0.18 A_{t},
\end{aligned}
$$

where $M_{i}(i=1,2,3)$ are the gaugino mass parameters. Note that all the parameters in the right-hand side are those evaluated at the GUT scale.

In eq. (2.2), we can see that the absolute value of $m_{H_{u}}\left(m_{Z}\right)$ becomes large, when the value of $m_{u_{3}}, m_{Q_{3}}$ or $A_{t}$ increases. Moreover, eqs. (2.3)-(2.5) tell us that the ratio 
$A_{t}\left(m_{Z}\right) / M_{\mathrm{st}}$ cannot be so large as far as the gluino mass $M_{3}$ dominates the RG corrections. Thus the naturalness and the $125 \mathrm{GeV}$ Higgs boson mass are hard to be achieved simultaneously.

Such a situation can be avoided by considering large values of wino and bino masses, particularly wino mass gives more significant contributions than the bino mass does. The contribution of the gluino mass $\left(M_{3}\right)$ to the RG running of $m_{H_{u}}\left(m_{Z}\right)$ can be canceled by those of the wino (and sub-dominantly bino) masses $\left(M_{2}\right.$ and $\left.M_{1}\right)$ as can be read off in eq. (2.2). More precisely, $m_{H_{u}}\left(m_{Z}\right)$ can remain small although top squark masses increase if the wino to gluino mass ratio at the GUT scale $\left(M_{2} / M_{3} \equiv r_{2}\right)$ is in the range $4 \lesssim r_{2} \lesssim 6[6]$.

The suitable ratio of wino to gluino mass does not only relax the degree of tuning, but also enhances the Higgs boson mass due to a relatively large value of the A-term. In eqs. (2.3) and (2.4), we see that only the left-handed third-generation squark mass $m_{Q_{3}}\left(m_{Z}\right)$ increases when the wino mass increases, while the right-handed top squark mass $m_{u_{3}}\left(m_{Z}\right)$ does not. Furthermore, $m_{u_{3}}\left(m_{Z}\right)$ tend to be small according to the top Yukawa coupling, compared with the other sparticle masses. As a result, only the right-handed top squark is significantly lighter than the other sfermions. Besides, the absolute value of $A_{t}\left(m_{Z}\right)$ will become large when the wino (bino) mass increases, since gaugino masses contribute to RG runnings of A-terms with the same sign. Consequently, a large ratio of A-term to top squark mass scale $A_{t}\left(m_{Z}\right) / M_{\text {st }}$ can be accomplished, and then the Higgs boson mass can reach $125 \mathrm{GeV}$ even when the top squark mass is lighter than $1 \mathrm{TeV}$. Note that we assume the gaugino masses dominate the RG-runnings, in other words, A-terms and scalar masses are not extremely larger than the gaugino masses.

\section{Phenomenological features of NUGM scenario}

In our analysis, we assume the soft SUSY-breaking scalar masses, including the Higgs soft masses, are universal and A-terms are flavor-independent just for simplicity, because these parameters do not play essential roles in the NUGM scenario. The values at the GUT scale are denoted by $m_{0}$ and $A_{0}$ respectively. Thus there are six independent parameters in our analysis: three gaugino masses $M_{1}, M_{2}, M_{3}$, universal scalar mass $m_{0}$, flavor independent A-term $A_{0}$.

For simplicity, we fix $\tan \beta=15$ in our analysis, with which the contributions of the bottom Yukawa couplings are negligibly small. Furthermore, $m_{0}$ is fixed at $1 \mathrm{TeV}$, because our main interest is the gaugino mass dependence. In section $4, M_{2}$ and $A_{0}$ are tuned to realize $\mu=150 \mathrm{GeV}$ and $125 \leq m_{h}<126 \mathrm{GeV}$, and the parameter space of $\left(M_{1}, M_{3}\right)$ is scanned in our analysis. We use the softsusy-3.5.1 [19] to evaluate masses and mixings of sparticles and Higgs bosons.

First, let us discuss $M_{2}$ and $A_{0}$ for $\mu=150 \mathrm{GeV}$ and $125 \leq m_{h}<126 \mathrm{GeV}$. Figure 1 shows the sizes of $M_{2}$ (left panel) and $A_{0}$ (right panel) on the plane of $\left(M_{1}, M_{3}\right)$. We can see that typical values of $M_{2}$ and $A_{0}$ are around $4.0 \mathrm{TeV}$ and $-2.5 \mathrm{TeV}$. In the gray region, the lightest top squark becomes tachyonic, so $M_{1}$ and $M_{3}$ cannot be so small to achieve the small $\mu$ term. 

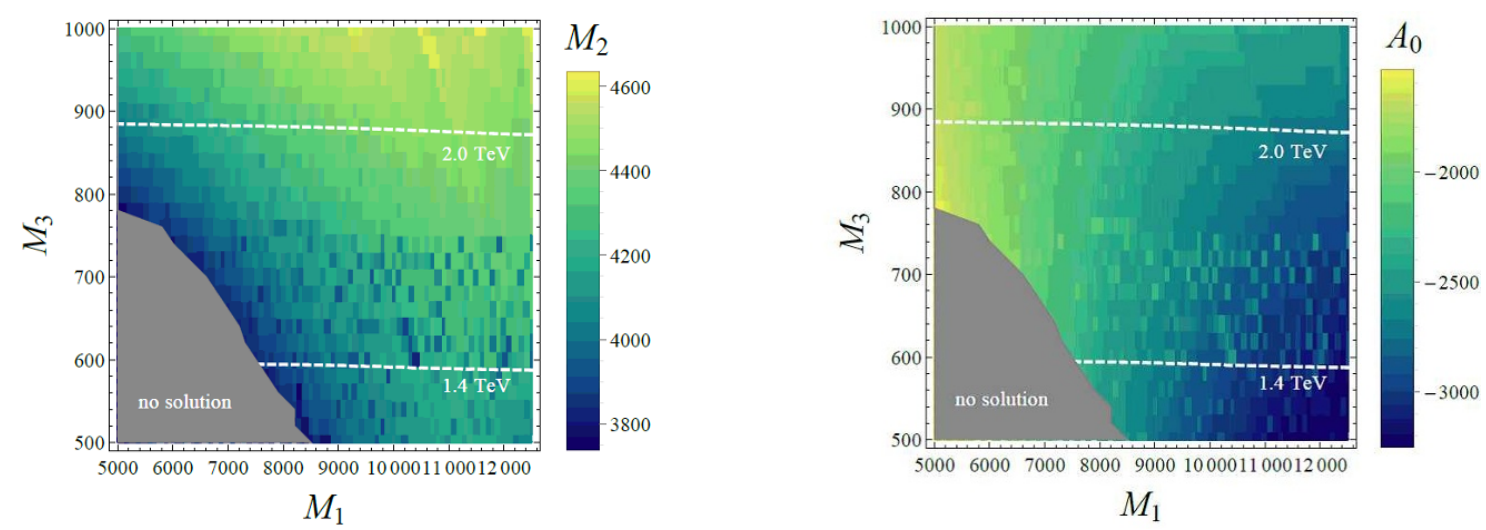

Figure 1. Values of input parameters, $M_{2}$ (left panel) and $A_{0}$ (right panel), at the GUT scale that achieve $\mu=150 \mathrm{GeV}$ and $125 \leq m_{h}<126 \mathrm{GeV}$. The white dashed lines represent the mass of the gluino at the EW scale. We cannot find input parameters that satisfy our requirements in the gray region. The values of $M_{1}, M_{2}, M_{3}$ and $A_{0}$ are shown in the unit $\mathrm{GeV}$.

Table 1 shows some benchmark points. We can see that the top squark $\widetilde{t}_{1}$ is significantly lighter than bottom squark $\widetilde{b}_{1}$ and gluino $\widetilde{g}$. Since the masses of bino and wino are around or larger than the gluino mass at the EW scale, then sleptons tend to be as heavy as squarks, other than the top squark, in contrast to the most of well-known scenarios, e.g. the CMSSM or the minimal gauge mediation [20].

In the NUGM, a top squark is lighter than gluino. Then the lower bound on the gluino mass will be given by the analysis in ref. [21] rather than the analysis in ref. [22]. In the former, the exclusion limit of the gluino mass reaches $1.4 \mathrm{TeV}$. This bound would depend on decay properties of the top squark, and then the exact bound on the NUGM is unknown. We will study gluino searches in the NUGM elsewhere, while in this paper we just expect that the bound on the gluino is around the result of ref. [21]. Then we concentrate on the parameter region of the NUGM with $m_{\widetilde{g}} \gtrsim 1.4 \mathrm{TeV}$ that corresponds to $M_{3} \gtrsim 600 \mathrm{GeV}$. Note that the other sparticles could not be produced at the LHC, since they are heavier than the gluino.

The masses of the lightest chargino $\widetilde{\chi}_{1}^{ \pm}$and the lightest and the second lightest neutralino $\widetilde{\chi}_{1,2}^{0}$ are just above $\mu=150 \mathrm{GeV}$, since they are virtually higgsino-like. In addition, the mixings among the higgsino-like states and bino- or wino-like states are highly suppressed, since $\widetilde{\chi}_{3,4}^{0}$, which are bino-like and wino-like neutralinos, are quite heavy. As a result, the mass differences between the higgsino-like states are highly suppressed.

The degenerate higgisnos will make $\widetilde{\chi}_{2}^{0}, \widetilde{\chi}_{1}^{ \pm}$decay into lighter higgsino-like states invisible. The left panel of figure 2 shows the mass difference between $\widetilde{\chi}_{1}^{0}$ and $\widetilde{\chi}_{1}^{ \pm}$. We can see that the mass difference is less than $2 \mathrm{GeV}$ and then their decay products would be lower than the reconstruction threshold. The mass difference between $\widetilde{\chi}_{2}^{0}$ and $\widetilde{\chi}_{1}^{ \pm}$is also less than $2 \mathrm{GeV}$.

The decay channels of the top squark are almost fixed in the NUGM. The top squark couples to neutralino and chargino through the top Yukawa coupling, and the lightest state is almost right-handed. Moreover, the masses of the higgsino-like states are nearly 


\begin{tabular}{|c|c|c|c|}
\hline input $[\mathrm{GeV}]$ & sample 1 & sample 2 & sample 3 \\
\hline$\mu\left(m_{Z}\right)$ & 150 & 150 & 150 \\
$m_{0}$ & 1000 & 1000 & 1000 \\
$A_{0}$ & -1950 & -2400 & -2500 \\
$M_{1}$ & 6500 & 9000 & 10000 \\
$M_{2}$ & 4231 & 4458 & 4478 \\
$M_{3}$ & 900 & 900 & 900 \\
\hline mass $[\mathrm{GeV}]$ & & & \\
\hline$m_{\widetilde{t}_{1}}$ & 695.2 & 1169 & 1414 \\
$m_{\widetilde{b}_{1}}$ & 1971 & 2081 & 2139 \\
$m_{\widetilde{g}}$ & 2035 & 2041 & 2046 \\
$m_{\widetilde{\chi}_{1}^{ \pm}}$ & 154.6 & 155.1 & 154.7 \\
$m_{\widetilde{\chi}_{1}^{0}}$ & 152.9 & 153.6 & 153.3 \\
$m_{\widetilde{\chi}_{2}^{0}}$ & 156.1 & 156.3 & 155.8 \\
$m_{\widetilde{\chi}_{3}^{0}}$ & 2884 & 3621 & 3639 \\
$m_{\widetilde{\chi}_{4}^{0}}$ & 3435 & 4004 & 4453 \\
\hline $\operatorname{branching}$ ratio & & & \\
\hline $\operatorname{Br}\left(\widetilde{t}_{1} \rightarrow t \widetilde{\chi}_{1}^{0}\right)$ & 0.228 & 0.242 & 0.245 \\
$\operatorname{Br}\left(\widetilde{t}_{1} \rightarrow t \widetilde{\chi}_{2}^{0}\right)$ & 0.240 & 0.249 & 0.251 \\
$\operatorname{Br}\left(\widetilde{t}_{1} \rightarrow b \widetilde{\chi}_{1}^{ \pm}\right)$ & 0.532 & 0.509 & 0.505 \\
$\operatorname{Br}\left(\widetilde{g} \rightarrow \widetilde{t}_{1} \tilde{t}\right)$ & 0.497 & 0.500 & 0.500 \\
\hline output & & & \\
\hline$N_{\text {signal }}$ & 5.04 & $3.71 \times 10^{-2}$ & $6.71 \times 10^{-3}$ \\
$S / \sqrt{B}$ & 43.2 & 5.41 & 1.71 \\
\hline
\end{tabular}

Table 1. Values of parameters at some sample points. $N_{\text {signal }}$ is the number of events coming from top squark pair production with the center of mass energy $\sqrt{s}=8 \mathrm{TeV}$ with an integrated luminosity $20.1 \mathrm{fb}^{-1}$ in a signal region with $m_{\mathrm{CT}}>350 \mathrm{GeV}$ of ref. [25]. $S / \sqrt{B}$ is the significance of the signal events, where $S$ and $B$ are the number of signal and background events respectively with a center of mass energy $\sqrt{s}=14 \mathrm{TeV}$ and an integrated luminosity of $300 \mathrm{fb}^{-1}$. For the numbers of backgrounds we refer to ref. [26], and then we select a signal region that maximizes the significance for each sample point.

degenerate as explained above. These facts fix the branching ratios of the top squark as,

$$
\begin{aligned}
& \operatorname{Br}\left(\widetilde{t}_{1} \rightarrow t \widetilde{\chi}_{1}^{0}\right) \simeq 25 \%, \\
& \operatorname{Br}\left(\widetilde{t}_{1} \rightarrow t \widetilde{\chi}_{2}^{0}\right) \simeq 25 \%, \\
& \operatorname{Br}\left(\widetilde{t}_{1} \rightarrow b \widetilde{\chi}_{1}^{ \pm}\right) \simeq 50 \% .
\end{aligned}
$$

The branching ratios, $\operatorname{Br}\left(\widetilde{t}_{1} \rightarrow b \widetilde{\chi}_{1}^{ \pm}\right) \equiv X$, are shown in the right panel of figure 2 . We use SDECAY [23] to compute the decay widths and the branching ratios of sparticles. The numerical values of $X$ significantly decrease around $\left(M_{1}, M_{3}\right) \sim(12,0.55) \mathrm{TeV}$ where the top squark can decay into or through the gluino. 

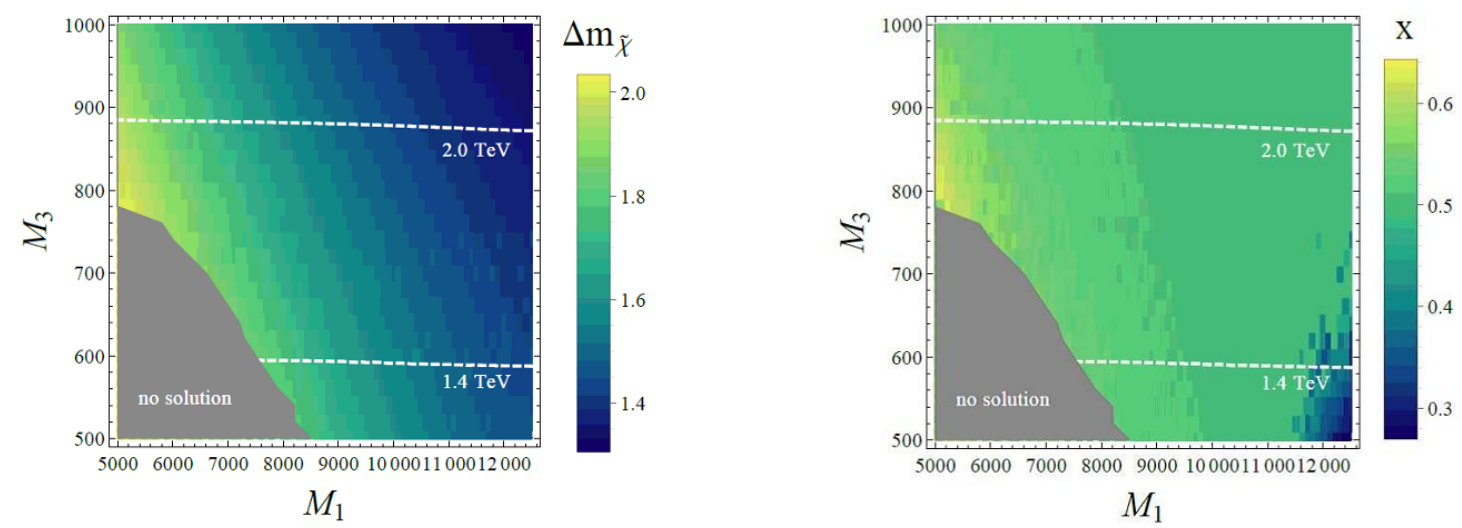

Figure 2. The mass difference between $\widetilde{\chi}_{1}^{ \pm}$and $\widetilde{\chi}_{1}^{0}$ (left panel) and the branching ratio of the top squark to the bottom quark and the lightest chargino (right panel), $X \equiv \operatorname{Br}\left(\widetilde{t}_{1} \rightarrow b \widetilde{\chi}_{1}^{ \pm}\right)$, are shown. The meanings of the lines and colored regions are the same as figure 1 . The values of $M_{1}, M_{3}$ and $\Delta m_{\tilde{\chi}}$ are shown in the unit $\mathrm{GeV}$.

\section{Top squark search at the LHC}

Finally, we discuss the signals of our top squark at the LHC. We use the Madgraph 5 [24] to simulate the signal events at the parton-level. After the event selections with suitable cuts, the number of events are compared with the $95 \%$ confidence level (C.L.) upper limits on the number of signal events given by the ATLAS collaboration [25]. We refer to table 11 of ref. [26] for the expected numbers of the background events at the LHC14. The higgsino-like states $\widetilde{\chi}_{1}^{ \pm}, \widetilde{\chi}_{1,2}^{0}$ are treated as invisible particles in our analysis, since the transverse momentum of SM particles produced by the higgsino decay would be below the reconstruction threshold.

\subsection{Details of the analysis}

We generate $10^{5}$ signal events at each point with $\sqrt{s}=8 \mathrm{TeV}$ or $14 \mathrm{TeV}$, then these are normalized to be consistent with the integrated luminosity that is observed or expected at the LHC, respectively.

Some properties of event reconstruction procedures are taken into account in our analysis with respect to ref. [25]. Electrons (muons) must have a transverse momentum, denoted as $p_{T}$, larger than $7(6) \mathrm{GeV}$ and their pseudo-rapidity must be in a range $|\eta|<2.47(2.4)$, otherwise leptons are discarded. Here a pseudo-rapidity is defined as $\eta \equiv-\log (\tan \theta / 2)$, where $\cos \theta \equiv p_{z} /|\boldsymbol{p}| .^{3}$ If quarks except top quarks have $p_{T}>20 \mathrm{GeV}$ and $|\eta|<4.9$, they are counted as jets. Then, if its flavor is bottom and $|\eta|<2.5$, it is interpreted as b-tagged jet with a b-tagging efficiency. We assume the b-tagging efficiency is $60 \%$ in our analysis. Finally, a missing transverse momentum $\boldsymbol{p}_{T}^{\text {miss }}$ of each event is defined as the one opposite to a sum of all visible particles within $|\eta|<4.9$, and then missing transverse energy is defined as $E_{T}^{\text {miss }} \equiv\left|\boldsymbol{p}_{T}^{\text {miss }}\right|$.

\footnotetext{
${ }^{3}$ The $\mathrm{z}$-axis is along the incident beam direction and $\boldsymbol{p}$ is a spatial momentum vector.
} 
If some of jets or leptons are overlapped in a $(\phi, \eta)$ plane, where $\phi$ is an azimuthal angle, they are resolved following the experimental analyses. The transverse momentum of two jets are summed and $\eta, \phi$ are summed weighted by each transverse momentum when the distance between two jets is within $\Delta R<0.4$, where $\Delta R \equiv \sqrt{(\Delta \eta)^{2}+(\Delta \phi)^{2}}$ is defined. If one of two jets is b-tagged, the jet after the resolving procedure is treated as a b-tagged jet. Overlaps between light-flavor jets and electrons within $\Delta R<0.2$ are resolved by discarding the jet, while the electron is discarded if the jet is b-tagged. When the overlaps between electrons (muons) and any jet are within $0.2<\Delta R<0.4(\Delta R<0.4)$, both of the electron and the muon are discarded.

\subsection{Results of the analysis}

Direct top squark searches have been done dedicated to the several channels at the ATLAS [25, 27-29] and the CMS [30-34].

Pair-produced top squarks decay into the SM particles in several ways. When a top squark decays into a top quark and a neutralino, $\tilde{t}_{1} \rightarrow t \tilde{\chi}_{1,2}^{0}$, the top quark decays into a bottom quark and a $\mathrm{W}$ boson which subsequently decays into two light-flavor quarks, or a charged lepton and a neutrino. Then the pair-produced top squarks decay as $\tilde{t}_{1} \tilde{t}_{1}^{*} \rightarrow$ $t \bar{t}+\tilde{\chi}^{0} \tilde{\chi}^{0} \rightarrow b \bar{b}+f \bar{f} f^{\prime} \bar{f}^{\prime}+\tilde{\chi}^{0} \tilde{\chi}^{0}$, where $f \bar{f}$ and $f^{\prime} \bar{f}^{\prime}$ are $q \bar{q}$ or $l \nu$. Thus corresponding channels are $2 b+(4 j$ or $2 j+1 l$ or $2 l)+E_{T}^{\text {miss }}$.

The $2 b+4 j+E_{T}^{\text {miss }}$ and $2 b+2 j+1 l+E_{T}^{\text {miss }}$ channels give more stringent bound on the top squark mass than the $2 b+2 l+E_{T}^{\text {miss }}$ channel, since the hadronic decay of $\mathrm{W}$ boson is about $70 \%$ although the leptonic channels would not be suffered from the SM background. In the $2 b+4 j+E_{T}^{\text {miss }}$ channel, one of the dominant SM backgrounds is the $t \bar{t}$ production, where one top quark decay semileptonically and the lepton is not reconstructed successfully, and the other is the $Z(\rightarrow \nu \nu)+$ jets [27]. In the $2 b+2 j+1 l+E_{T}^{\text {miss }}$ channel, the SM backgrounds are dominated by the $t \bar{t}$ production, where the both top quarks decays semileptonically and one lepton is not reconstructed, and the $W+$ jets where the $\mathrm{W}$ boson decays leptonically [28].

When a top squark decays into a bottom quark and a chargino, $\tilde{t}_{1} \rightarrow b \tilde{\chi}_{1}^{ \pm}$, the chargino decays into the lightest neutralino and two light-flavor quarks or a charged lepton and a neutrino, where the two SM fermions are produced through the off-shell W boson if the mass difference between the neutralino and the chargino is less than the $\mathrm{W}$ boson mass. Then the pair-produced top squarks decay as, $\tilde{t}_{1} \tilde{t}_{1}^{*} \rightarrow b \bar{b}+\tilde{\chi}_{1}^{+} \tilde{\chi}_{1}^{-} \rightarrow b \bar{b}+f \bar{f} f^{\prime} \bar{f}^{\prime}+\tilde{\chi}^{0} \tilde{\chi}^{0}$. The signal is similar to the case of $\tilde{t}_{1} \tilde{t}_{1}^{*} \rightarrow t \bar{t}+\tilde{\chi}^{0} \tilde{\chi}^{0}$. However the daughter particles of the chargino become soft due to the small mass difference between the neutralino and the chargino, and then soft leptons are required by the event selections in many analyses targeting to the top squarks decaying into bottom quarks and charginos.

Such soft daughter particles of the chargino could be too soft to be reconstructed at the detector if the mass difference between the neutralino and the chargino is quite small. This occurs exactly in the NUGM scenario, since their mass difference is smaller than $2 \mathrm{GeV}$. Thus the corresponding channel to this case is $2 b+E_{T}^{\text {miss }}$ where all of the daughter particles of the chargino are not reconstructed. In this channel, the dominant SM background is $Z(\rightarrow \nu \nu)+$ jets where jets should include b-jets, and the backgrounds coming from top quarks will become sub-dominant [25]. 


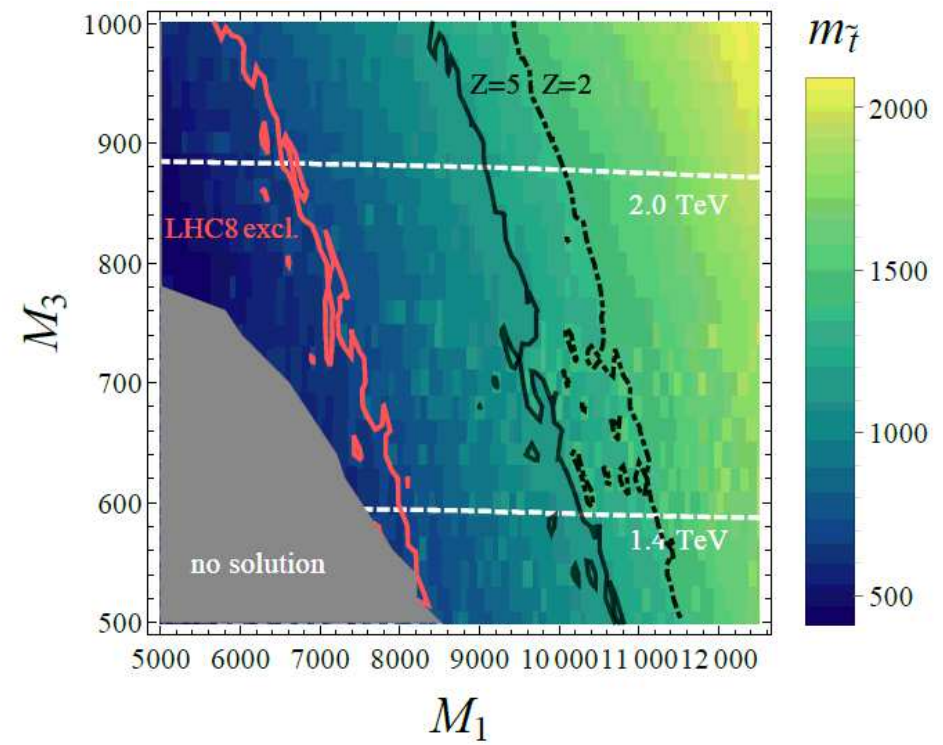

Figure 3. Masses of the top squark and its experimental bound. The red line is the 95\% C.L. limits from the data with $\sqrt{s}=8 \mathrm{TeV}$ and an integrated luminosity of $20.1 \mathrm{fb}^{-1}$. The black lines represent the expected significance $Z \equiv S / \sqrt{B}$ with $\sqrt{s}=14 \mathrm{TeV}$ and an integrated luminosity of $300 \mathrm{fb}^{-1}$. The meanings of the white lines and colored regions are the same as figure 1 . The values of $M_{1}, M_{3}$ and $m_{\tilde{t}}$ are shown in the unit $\mathrm{GeV}$.

Since the branching ratio of $\widetilde{t}_{1} \rightarrow t \widetilde{\chi}_{1,2}^{0}$ is around $50 \%$, the analyses dedicated to the channels $t \bar{t}+E_{T}^{\text {miss }}$ may not be so efficient in the NUGM scenario. The corresponding number of signal events is quarter, compared with the case with $\operatorname{Br}\left(\widetilde{t}_{1} \rightarrow t \widetilde{\chi}_{1,2}^{0}\right)=100 \%$. This is almost same as the number of signal events in the $2 b+E_{T}^{\text {miss }}$ channel. Thus the most efficient channel to investigate the NUGM scenario would be $2 b+E_{T}^{\text {miss }}$, because the SM background from $Z(\rightarrow \nu \nu)+$ jets would be easier to be distinguished from the signal events than those of $t \bar{t}$ production. These arguments will depend on details of analyses, but these observations are also pointed out and confirmed in ref. [35].

The $95 \%$ C.L. upper limit on the number of the SUSY event is displayed in table 7 of ref. [25] that is analyses based on the data with $\sqrt{s}=8 \mathrm{TeV}$ and the integrated luminosity of $20.1 \mathrm{fb}^{-1}$. The number of signal events after the same event selection criteria is calculated following the method explained in the previous subsection. The number of survived events at the sample points are shown in table 1.

The expected number of the background events dedicated to $2 b+E_{T}^{\text {miss }}$ with $\sqrt{s}=$ $14 \mathrm{TeV}$ and with an integrated luminosity of $300 \mathrm{fb}^{-1}$ is studied in ref. [26]. The number of events can be seen in table 11 of ref. [26]. In fact, this analysis is devoted to the search for the bottom squark, through the decay as $\widetilde{b}_{1} \rightarrow b \widetilde{\chi}_{1}^{0}$. However, the expected final states and their kinematics are quite similar to $\widetilde{t}_{1} \rightarrow b \widetilde{\chi}_{1}^{ \pm}$, if the chargino $\widetilde{\chi}_{1}^{ \pm}$can be treated as the invisible particle effectively. The expected significance $Z \equiv S / \sqrt{B}$, where $S$ is the number of signal events after the event selection following the analysis in ref. [26], is shown in the table 1. 
Figure 3 shows the top squark mass and the experimental limits obtained from our analyses. In this figure, the red line describes the $95 \%$ C.L. exclusion upper limit obtained from the data with $\sqrt{s}=8 \mathrm{TeV}$ and the black lines represent the significance $Z \equiv S / \sqrt{B}=$ 2 (dashed) and 5 (solid). There are several signal regions corresponding to different cut with respect to the values of the contransverse mass $m_{\mathrm{CT}}$. The most sensitive signal region is selected at each point.

We can see that the current experimental lower bounds on the top squark mass in the NUGM is about $690 \mathrm{GeV}$. The bounds on the gaugino masses are $M_{1} \gtrsim 6.0-8.0 \mathrm{TeV}$ depending on the value of $M_{3}$. The top squark mass is mostly determined by $M_{1}$ in our analysis. Consequently the top squark mass is sensitive to $M_{3}$ in general, but its contribution is subtracted by those of $M_{2}$ in the NUGM scenario according to the constraint on the small $\mu$ term, or equivalently small $\left|m_{H_{u}}\right|$. The top squark mass is sensitive to $M_{1}$, while $m_{H_{u}}$ is relatively insensitive to it [5].

Figure 3 also shows the expected sensitivity at the LHC14 with an integrated luminosity of $300 \mathrm{fb}^{-1}$. It is found that the significance reaches $Z=5$ that maybe correspond to the discovery for $m_{\widetilde{t}_{1}} \simeq 1.2 \mathrm{TeV}$, and reaches $Z=2$ that may correspond to an exclusion limit for $m_{\widetilde{t}_{1}} \simeq 1.4 \mathrm{TeV}$.

\section{Conclusion}

In this paper, we have studied phenomenological properties relevant to collider experiments of the non-universal gaugino masses (NUGM) scenario. Especially, we have discussed the current and expected sensitivities of the top squark searches at the LHC.

The NUGM scenario can relax the fine-tuning of the $\mu$-parameter [5], while can enhance the SM-like Higgs boson mass through the RG-runnings [6]. The key ingredient of the NUGM is a ratio of the wino to gluino mass, $r_{2} \equiv M_{2} / M_{3}$, at the GUT scale which should be in a range $4 \lesssim r_{2} \lesssim 6[5,6]$. In this case, the lightest top squark, which is almost right-handed one, is lighter than all the other sfermions and gauginos.

The gaugino mass ratios should be fixed precisely in order to avoid the fine-tuning, then we have to pay attention to how the desirable ratios are realized. The NUGM scenario can be obtained from some UV physics. An interesting possibility is the TeV-scale mirage mediation [36-40], that is, a mixed SUSY breaking mediation via the moduli and the conformal anomaly [41, 42]. The desired cancellation among the gaugino masses in RG runnings occurs, even when the so-called mirage unification relation does not hold exactly, or there are gauge mediated contributions in addition to the mirage mediation [43] if it has suitable values [44]. The gaugino masses are controlled by the ratio of the contributions from moduli and anomaly mediation, which depends on the moduli stabilization scenarios [45].

The non-universal gaugino masses can also be realized even at the tree-level (in fourdimensional effective field theory). For example, certain linear-combinations of multiple muduli fields appear in the gauge kinetic functions in some superstring models with nontrivial D-brane configurations, and then gaugino mass ratios are determined by e.g. the numbers of winding, intersection or magnetic fluxes for D-branes from which the SM gauge bosons arises [46]. In the case that the gauge boson for each SM gauge group lives on 
a different D-brane from each other, their gauge kinetic functions are different linearcombinations, that could be the origin of gaugino mass non-universality. Furthermore, the $\mathrm{U}(1)_{Y}$ symmetry of the SM may be given by a linear combination of multiple $\mathrm{U}(1)$ symmetries with quite different origins. This yields a correction to the bino mass of its own. Finally, even in the four-dimensional SUSY GUT framework, SUSY breaking fields contained in the gauge kinetic function may not be singlet under the GUT symmetry. They will give non-universal masses to gauginos depending on their representations [47].

Let us comment on dark matter candidates in the NUGM scenario. The LSP is typically purely higgsino-like and its mass is $\mathcal{O}(100 \mathrm{GeV})$. This means the amount of thermally produced LSP is quite less than the cosmologically required value [48]. The relic density can be accomplished when new dark matter candidate is introduced in addition to the LSP, such as axions, and/or there is an enough amount of non-thermally produced LSP comes from decays of long-lived heavy particles such as gravitino [49] and/or modulus fields [50]. The collider physics would be unchanged in these cases. Even when we consider thermally produced dark matter composed of only the LSP, the NUGM scenario can also provide the suitable dark matter candidate. One way is that LSP is mixture of higgsino and bino that can be achieved in the parameter region $M_{1} \ll M_{2}, M_{3}$ although such region is out of the figures in this paper [51]. Another possibility is to introduce new sparticle lighter than the neutralinos in extended models of the MSSM. Interesting candidates are an axino which is a superpartner of axion [52-54], or the singlino in the Next-to-MSSM [55]. In these cases, the collider phenomenology would be altered from the analyses in this paper.

Since the mass differences between the lightest neutralino and the lightest chargino $\Delta m_{\widetilde{\chi}_{1}^{ \pm}-\widetilde{\chi}_{1}^{0}}$, or the second-lightest neutralino and the lightest chargino $\Delta m_{\widetilde{\chi}_{2}^{0}-\widetilde{\chi}_{1}^{ \pm}}$are typically less than $2 \mathrm{GeV}$, all of decay products of the heavier higgsino-like states are invisible. The lightest top squark decays into $t \widetilde{\chi}^{0}$ and $b \widetilde{\chi}^{ \pm}$with almost the same branching ratio, because the top squark is made of mostly the right-handed one. These features make it difficult to search for top squark production dedicated to $t \bar{t}+E_{T}^{\text {miss }}$ or $b \bar{b}+f \bar{f} f^{\prime} \bar{f}^{\prime}+E_{T}^{\text {miss }}$. Thus the $b \bar{b}+E_{T}^{\text {miss }}$ channel gives the most stringent bound on the top squark mass.

We find out the experimental $95 \%$ C.L. exclusion limit on the NUGM by referring to the result of the analysis dedicated to $b \bar{b}+E_{T}^{\text {miss }}$ channel with $\sqrt{s}=8 \mathrm{TeV}$ data and an integrated luminosity of $20.1 \mathrm{fb}^{-1}$. The lower bound on the top squark mass is about $690 \mathrm{GeV}$ and the allowed region on the $\left(M_{1}, M_{3}\right)$ plane can be shown in figure 3.

We also studied the expected significance at the LHC with $\sqrt{s}=14 \mathrm{TeV}$ and $300 \mathrm{fb}^{-1}$. The significance of the signal of the $b \bar{b}+E_{T}^{\text {miss }}$ channel will reach $Z=5$ if the lightest top squark mass is less than $1.2 \mathrm{TeV}$ and will reach $Z=2$ if the mass is less than $1.4 \mathrm{TeV}$ as can be seen in figure 3 . Thus the top squark lighter than a mass scale around $1.2 \mathrm{TeV}$ will be discovered and the top squark lighter than about $1.4 \mathrm{TeV}$ could be excluded.

Finally, let us comment on the other possibilities to probe the NUGM scenario. Firstly, a single top quark channel, $t b+E_{T}^{\text {miss }}$, would be promising to probe the NUGM, since the half of the decay from the top squark corresponds to the signal, $t b+E_{T}^{\text {miss }}$, due to the branching ratio of the top squark, $\operatorname{Br}\left(\widetilde{t}_{1} \rightarrow t \widetilde{\chi}_{1,2}^{0}\right) \simeq \operatorname{Br}\left(\widetilde{t}_{1} \rightarrow b \widetilde{\chi}_{1}^{ \pm}\right) \simeq 50 \%$. Thus this channel could give the same or even severer limits on the top squark masses. The top squark becomes light in the small $M_{1}$ region, while the gluino search will become the most impor- 
tant in the small $M_{3}$ (and large $M_{1}$ ) region. Since the gluino will decay into the top squark in the latter region, then the features of the top squark decays showed in this paper will also be important for the gluino searches. We can also consider the case where $\tan \beta$ is so large that the bottom Yukawa coupling becomes the same order as the top one. In such case, the right-handed bottom squark would be lighter than the other sfermions, then the bottom squark becomes also accessible at the LHC. We will study these possibilities in the future.

\section{Acknowledgments}

This work was supported in part by the Grant-in-Aid for Scientific Research No. 25800158 (H.A.) and No. 23104011 (Y.O.) from the Ministry of Education, Culture, Sports, Science and Technology (MEXT) in Japan.

Open Access. This article is distributed under the terms of the Creative Commons Attribution License (CC-BY 4.0), which permits any use, distribution and reproduction in any medium, provided the original author(s) and source are credited.

\section{References}

[1] S.P. Martin, A supersymmetry primer, in Perspectives on supersymmetry II, G.L. Kane ed., World Scientific, Singapore (2010), pg. 1 [Adv. Ser. Direct. High Energy Phys. 21 (2010) 1] [hep-ph/9709356] [INSPIRE].

[2] ATLAS collaboration, Observation of a new particle in the search for the standard model Higgs boson with the ATLAS detector at the LHC, Phys. Lett. B 716 (2012) 1 [arXiv: 1207.7214] [INSPIRE].

[3] CMS collaboration, Observation of a new boson at a mass of $125 \mathrm{GeV}$ with the CMS experiment at the LHC, Phys. Lett. B 716 (2012) 30 [arXiv:1207.7235] [INSPIRE].

[4] R. Barbieri and G.F. Giudice, Upper bounds on supersymmetric particle masses, Nucl. Phys. B 306 (1988) 63 [INSPIRE].

[5] H. Abe, T. Kobayashi and Y. Omura, Relaxed fine-tuning in models with non-universal gaugino masses, Phys. Rev. D 76 (2007) 015002 [hep-ph/0703044] [INSPIRE].

[6] H. Abe, J. Kawamura and H. Otsuka, The Higgs boson mass in a natural MSSM with nonuniversal gaugino masses at the GUT scale,

Prog. Theor. Exp. Phys. 2013 (2013) 013B02 [arXiv: 1208.5328] [InSPIRE].

[7] ATLAS collaboration, Search for direct production of charginos, neutralinos and sleptons in final states with two leptons and missing transverse momentum in pp collisions at $\sqrt{s}=8 \mathrm{TeV}$ with the ATLAS detector, JHEP 05 (2014) 071 [arXiv:1403.5294] [INSPIRE].

[8] ATLAS collaboration, Search for direct production of charginos and neutralinos in events with three leptons and missing transverse momentum in $\sqrt{s}=8 \mathrm{TeV} p p$ collisions with the ATLAS detector, JHEP 04 (2014) 169 [arXiv: 1402.7029] [INSPIRE].

[9] CMS collaboration, Searches for electroweak production of charginos, neutralinos and sleptons decaying to leptons and $W, Z$ and Higgs bosons in pp collisions at $8 \mathrm{TeV}$, Eur. Phys. J. C 74 (2014) 3036 [arXiv: 1405.7570] [INSPIRE]. 
[10] H. Baer et al., Radiatively-driven natural supersymmetry at the LHC, JHEP 12 (2013) 013 [Erratum ibid. 06 (2015) 053] [arXiv:1310.4858] [INSPIRE].

[11] H. Baer, A. Mustafayev and X. Tata, Monojet plus soft dilepton signal from light higgsino pair production at LHC14, Phys. Rev. D 90 (2014) 115007 [arXiv:1409.7058] [INSPIRE].

[12] H. Baer et al., Same sign diboson signature from supersymmetry models with light higgsinos at the LHC, Phys. Rev. Lett. 110 (2013) 151801 [arXiv:1302.5816] [INSPIRE].

[13] H. Baer, V. Barger, P. Huang, D. Mickelson, A. Mustafayev and X. Tata, Radiative natural supersymmetry: reconciling electroweak fine-tuning and the Higgs boson mass,

Phys. Rev. D 87 (2013) 115028 [arXiv:1212.2655] [INSPIRE].

[14] C. Han, A. Kobakhidze, N. Liu, A. Saavedra, L. Wu and J.M. Yang, Probing light higgsinos in natural SUSY from monojet signals at the LHC, JHEP 02 (2014) 049 [arXiv:1310.4274] [INSPIRE].

[15] ATLAS collaboration, Search for charginos nearly mass degenerate with the lightest neutralino based on a disappearing-track signature in pp collisions at $\sqrt{s}=8 \mathrm{TeV}$ with the ATLAS detector, Phys. Rev. D 88 (2013) 112006 [arXiv:1310.3675] [INSPIRE].

[16] CMS collaboration, Search for disappearing tracks in proton-proton collisions at $\sqrt{s}=8 \mathrm{TeV}, \mathrm{JHEP} 01$ (2015) 096 [arXiv:1411.6006] [INSPIRE].

[17] M. Carena, M. Quirós and C.E.M. Wagner, Effective potential methods and the Higgs mass spectrum in the MSSM, Nucl. Phys. B 461 (1996) 407 [hep-ph/9508343] [InSPIRE].

[18] J.L. Feng, K.T. Matchev and T. Moroi, Focus points and naturalness in supersymmetry, Phys. Rev. D 61 (2000) 075005 [hep-ph/9909334] [INSPIRE].

[19] B.C. Allanach, SOFTSUSY: a program for calculating supersymmetric spectra, Comput. Phys. Commun. 143 (2002) 305 [hep-ph/0104145] [INSPIRE].

[20] G.F. Giudice and R. Rattazzi, Theories with gauge mediated supersymmetry breaking, Phys. Rept. 322 (1999) 419 [hep-ph/9801271] [InSPIRE].

[21] ATLAS collaboration, Search for strong production of supersymmetric particles in final states with missing transverse momentum and at least three b-jets at $\sqrt{s}=8 \mathrm{TeV}$ proton-proton collisions with the ATLAS detector, JHEP 10 (2014) 24 [arXiv:1407.0600] [INSPIRE].

[22] ATLAS collaboration, Search for squarks and gluinos with the ATLAS detector in final states with jets and missing transverse momentum using $\sqrt{s}=8$ TeV proton-proton collision data, JHEP 09 (2014) 176 [arXiv:1405.7875] [INSPIRE].

[23] M. Muhlleitner, A. Djouadi and Y. Mambrini, SDECAY: a fortran code for the decays of the supersymmetric particles in the MSSM, Comput. Phys. Commun. 168 (2005) 46 [hep-ph/0311167] [INSPIRE].

[24] J. Alwall et al., The automated computation of tree-level and next-to-leading order differential cross sections and their matching to parton shower simulations, JHEP 07 (2014) 079 [arXiv:1405.0301] [INSPIRE].

[25] ATLAS collaboration, Search for direct third-generation squark pair production in final states with missing transverse momentum and two b-jets in $\sqrt{s}=8$ TeV pp collisions with the ATLAS detector, JHEP 10 (2013) 189 [arXiv:1308.2631] [INSPIRE]. 
[26] ATLAS collaboration, Search for supersymmetry at the high luminosity LHC with the ATLAS experiment, ATL-PHYS-PUB-2014-010, CERN, Geneva Switzerland (2014).

[27] ATLAS collaboration, Search for direct pair production of the top squark in all-hadronic final states in proton-proton collisions at $\sqrt{s}=8 \mathrm{TeV}$ with the ATLAS detector, JHEP 09 (2014) 015 [arXiv: 1406.1122] [INSPIRE].

[28] ATLAS collaboration, Search for top squark pair production in final states with one isolated lepton, jets and missing transverse momentum in $\sqrt{s}=8 \mathrm{TeV}$ pp collisions with the ATLAS detector, JHEP 11 (2014) 118 [arXiv:1407.0583] [INSPIRE].

[29] ATLAS collaboration, Search for direct top-squark pair production in final states with two leptons in pp collisions at $\sqrt{s}=8 \mathrm{TeV}$ with the ATLAS detector, JHEP 06 (2014) 124 [arXiv: 1403.4853] [INSPIRE].

[30] CMS collaboration, Searches for third-generation squark production in fully hadronic final states in proton-proton collisions at $\sqrt{s}=8 \mathrm{TeV}$, JHEP 06 (2015) 116 [arXiv:1503.08037] [INSPIRE].

[31] CMS collaboration, Searches for supersymmetry using the $M_{T 2}$ variable in hadronic events produced in pp collisions at $8 \mathrm{TeV}$, JHEP 05 (2015) 078 [arXiv: 1502.04358] [INSPIRE].

[32] CMS collaboration, Search for supersymmetry using razor variables in events with b-tagged jets in pp collisions at $\sqrt{s}=8 \mathrm{TeV}$, Phys. Rev. D 91 (2015) 052018 [arXiv:1502.00300] [INSPIRE].

[33] CMS collaboration, Search for physics beyond the standard model in events with two leptons, jets and missing transverse momentum in pp collisions at $\sqrt{s}=8 \mathrm{TeV}$, JHEP 04 (2015) 124 [arXiv: 1502.06031] [INSPIRE].

[34] CMS collaboration, Search for top-squark pair production in the single-lepton final state in pp collisions at $\sqrt{s}=8 \mathrm{TeV}$, Eur. Phys. J. C 73 (2013) 2677 [arXiv:1308.1586] [INSPIRE].

[35] C. Han, K.-I. Hikasa, L. Wu, J.M. Yang and Y. Zhang, Current experimental bounds on stop mass in natural SUSY, JHEP 10 (2013) 216 [arXiv:1308.5307] [INSPIRE].

[36] M. Endo, M. Yamaguchi and K. Yoshioka, A bottom-up approach to moduli dynamics in heavy gravitino scenario: superpotential, soft terms and sparticle mass spectrum, Phys. Rev. D 72 (2005) 015004 [hep-ph/0504036] [INSPIRE].

[37] K. Choi, K.S. Jeong and K.-I. Okumura, Phenomenology of mixed modulus-anomaly mediation in fluxed string compactifications and brane models, JHEP 09 (2005) 039 [hep-ph/0504037] [INSPIRE].

[38] K. Choi, K.S. Jeong, T. Kobayashi and K.-I. Okumura, Little SUSY hierarchy in mixed modulus-anomaly mediation, Phys. Lett. B 633 (2006) 355 [hep-ph/0508029] [INSPIRE].

[39] K. Choi, K.S. Jeong, T. Kobayashi and K.-I. Okumura, TeV scale mirage mediation and natural little SUSY hierarchy, Phys. Rev. D 75 (2007) 095012 [hep-ph/0612258] [INSPIRE].

[40] R. Kitano and Y. Nomura, A solution to the supersymmetric fine-tuning problem within the MSSM, Phys. Lett. B 631 (2005) 58 [hep-ph/0509039] [INSPIRE].

[41] L. Randall and R. Sundrum, Out of this world supersymmetry breaking, Nucl. Phys. B 557 (1999) 79 [hep-th/9810155] [INSPIRE]. 
[42] G.F. Giudice, M.A. Luty, H. Murayama and R. Rattazzi, Gaugino mass without singlets, JHEP 12 (1998) 027 [hep-ph/9810442] [INSPIRE].

[43] L.L. Everett, I.-W. Kim, P. Ouyang and K.M. Zurek, Deflected mirage mediation: a framework for generalized supersymmetry breaking, Phys. Rev. Lett. 101 (2008) 101803 [arXiv: 0804.0592] [INSPIRE].

[44] H. Abe and J. Kawamura, The $126 \mathrm{GeV}$ Higgs boson mass and naturalness in (deflected) mirage mediation, JHEP 07 (2014) 077 [arXiv: 1405.0779] [INSPIRE].

[45] H. Abe, T. Higaki and T. Kobayashi, KKLT type models with moduli-mixing superpotential, Phys. Rev. D 73 (2006) 046005 [hep-th/0511160] [INSPIRE].

[46] R. Blumenhagen, B. Körs, D. Lüst and S. Stieberger, Four-dimensional string compactifications with D-branes, orientifolds and fluxes, Phys. Rept. 445 (2007) 1 [hep-th/0610327] [INSPIRE].

[47] J.E. Younkin and S.P. Martin, Non-universal gaugino masses, the supersymmetric little hierarchy problem and dark matter, Phys. Rev. D 85 (2012) 055028 [arXiv:1201.2989] [INSPIRE].

[48] S. Mizuta and M. Yamaguchi, Coannihilation effects and relic abundance of Higgsino dominant LSPs, Phys. Lett. B 298 (1993) 120 [hep-ph/9208251] [INSPIRE].

[49] K. Kohri, M. Yamaguchi and J. Yokoyama, Neutralino dark matter from heavy gravitino decay, Phys. Rev. D 72 (2005) 083510 [hep-ph/0502211] [INSPIRE].

[50] T. Moroi and L. Randall, Wino cold dark matter from anomaly mediated SUSY breaking, Nucl. Phys. B 570 (2000) 455 [hep-ph/9906527] [INSPIRE].

[51] N. Arkani-Hamed, A. Delgado and G.F. Giudice, The well-tempered neutralino, Nucl. Phys. B 741 (2006) 108 [hep-ph/0601041] [INSPIRE].

[52] K. Rajagopal, M.S. Turner and F. Wilczek, Cosmological implications of axinos, Nucl. Phys. B 358 (1991) 447 [inSPIRE].

[53] L. Covi, J.E. Kim and L. Roszkowski, Axinos as cold dark matter, Phys. Rev. Lett. 82 (1999) 4180 [hep-ph/9905212] [INSPIRE].

[54] L. Covi, H.-B. Kim, J.E. Kim and L. Roszkowski, Axinos as dark matter, JHEP 05 (2001) 033 [hep-ph/0101009] [INSPIRE].

[55] U. Ellwanger, C. Hugonie and A.M. Teixeira, The next-to-minimal supersymmetric standard model, Phys. Rept. 496 (2010) 1 [arXiv:0910.1785] [INSPIRE]. 